\title{
Influencing Factors of Intention and Behavior of Patient Delay in Elderly Benign Prostatic Hyperplasia: a Descriptive Qualitative Study
}

Hao Zou

Harbin Medical University (Daqing)

Dong-xu Jiang

Harbin Medical University (Daqing)

Wan-yue Zhao

Harbin Medical University (Daqing)

Hong-hong Jia

Harbin Medical University (Daqing)

Ying-li Li

Harbin Medical University (Daqing)

Jin-hong Yang

People's Hospital in Hei Longjiang Province

Lin-lin Zhang ( $\nabla$ zhanglinlin1971@163.com )

Changzhou University

\section{Research Article}

Keywords: Benign prostatic hyperplasia, Patient delay, Intention, qualitative study

Posted Date: January 6th, 2022

DOI: https://doi.org/10.21203/rs.3.rs-1155453/v1

License: (9) This work is licensed under a Creative Commons Attribution 4.0 International License.

Read Full License 


\section{Abstract}

Background: Most elderly patients with BPH have experienced patient delay. Based on the TPB, the aim of this study was to describe the influencing factors of BPH patients' intention and behavior of patient delay and to provide a reference for the development of a patient delay intention scale and personalized intervention.

Methods: This descriptive qualitative study was carried out 4 months in 2021 in China. The participants were 20 patients with BPH ages 60 to 82 years old who experienced patient delay and were selected through purposive sampling method. The study was performed in a large general hospital and a community hospital. The data were collected via face-to-face semi-structured interviews.

Results: The following themes were relevant to BPH patients with intention and behavior of patient delay: "Insufficient cognition of symptoms (low symptom alertness, symptom benign attribution)", "Experience coping instead of going to a doctor", "Negative attitude toward seeking medical care (Underestimation of the consequences of delayed medical treatment, Negative evaluation of early medical treatment)", "The influence of others on decision-making for seeking medical care(People who approve or disapprove of going to a doctor, Do or do not follow the opinions of others)", "Obstacles to seeking medical care(Factors from the patient, Factors from medical institutions)"

Conclusion: The delay was longer in rural areas than in urban areas. BPH patients' intention and behavior of patient delay are the result of a combination of many factors. Therefore, it is recommended to develop a scale to evaluate the patient delay intention for $\mathrm{BPH}$, and provide personalized interventions based on the scale to improve patient delay in elderly $\mathrm{BPH}$ patients

\section{Background}

Benign prostatic hyperplasia (BPH) is an age-related disease that mainly manifests as lower urinary tract symptoms (LUTS), such as urgency, frequency, nocturia incomplete urination, and weak urinary stream [1]. It is estimated that there will be 75 million elderly BPH patients in China in 2025 [2]. In the US, BPHrelated LUTS affects more than $20 \%$ of men aged $30-79$, approximately 15 million total [3]. A metaanalysis of the global prevalence of BPH reports that the lifetime prevalence of BPH is $26.2 \%$; that is, nearly $1 / 4$ of men will suffer from BPH during their lifetimes [1]. Studies have shown that more than $50 \%$ of elderly BPH patients experience patient delay [4]. Delayed treatment of patients with moderate or severe LUTS may induce serious complications such as hematuria, acute urinary retention, hydronephrosis, and renal failure [5], which will increase the burden of treatment for patients. The UK spends more than $£ 1.8$ million on the treatment of BPH each year, with approximately $60 \%$ of these costs incurred in secondary care as a direct result of managing complications [6]. How to improve patient delay in elderly BPH patients and reduce the burden of disease is an urgent problem that needs to be solved. Especially in China, low attention is given to the health management of $\mathrm{BPH}$. 
Patient delay refers to the time between a patient first observing suspicious symptoms and an interval of at least 3 months passing before the first visit to a medical institution [7]. The occurrence of this behavior is affected by some objective and non-objective factors. The theory of planned behavior (TPB) explains the general decision-making process of individual behavior from the perspective of information processing, and starting from the theory of expected value, the TPB is widely used in the study of health care seeking behavior [8-10]. According to the TPB, behavioral intentions are the most proximal determinant of behavior, and behavioral intention is determined by three factors: negative or positive evaluation of behavior (attitude), perception of attitudes toward others or society in the course of performing the behavior (subjective norm), and perception of the difficulty of completing the behavior (perceived control behavior) [11-12]. Moreover, Ajzen suggested that the TPB was open to interpretation, and researchers can expand the original model by adding new predictors to further increase the explanatory power toward behavioral intention and behavior [13]. Therefore, based on the TPB, this study adopted the descriptive qualitative study method to describe the influencing factors of BPH patients' intention and behavior of patient delay and to provide a reference for the development of a patient delay intention scale and personalized intervention.

\section{Material And Methods}

\section{Sample}

This research adopted the purposive sampling method, and 20 elderly BPH patients ( $\geq 60$ years) who experienced patient delay were sampled with maximum variation in a large general hospital and a community hospital in Daqing. Patients who were mentally ill or too serious to cooperate with the interview were excluded. All patients met the diagnostic criteria for patient delay and BPH.

\section{Date collection}

Data were collected via face-to-face semi-structured interviews from June to September 2021. Participants' demographic characteristics and IPSS scores (to evaluate the severity of LUTS) were collected before the formal interview. The interview time and place were agreed upon by the patient in advance. The interviews lasted 30-60 min until data saturation, i.e., no new data were obtained from the interviews. When the interviewee's responses were vague, we used skills such as questioning, repetition, and summarization to encourage interviewees to fully express their situation and ideas. With the consent of the interviewee, the interviews were audio recorded with a voice recorder. All recordings were managed confidentially.

After referring to similar literature, the research group discussed and combined with the TPB to draft a preliminary interview outline. Three patients were selected for pre-interview, which was revised again according to the interview results to form a formal interview outline. During the interview, the outline was constantly revised according to the interview data. For example, when a patient mentioned using folk 
prescriptions to relieve symptoms, "How do you deal with these symptoms before going to the doctor?", was added in the following interview to determine whether this situation was common among patients. The final interview outline was as follows (Table 1):

\section{Table 1}

Outline of the interview

outline of the interview

1. What symptoms did you have before you went to the doctor? Can you tell me what you think about these symptoms?

- Can you talk about how you dealt with these symptoms before you went to the doctor?

- Why were these measures being taken? How did you know about these measures?

2. Did you think there will be any consequences for the delay in seeing a doctor for this disease? What did you think of these consequences?

3. Can you talk about the reasons or events that didn't make you want to go to the doctor earlier?

4. Which people or situations will affect your decision to seek a doctor?

- Can you tell me what you think of their views, opinions, or behavior?

- What did you do with their opinions? Why did you do that? What did you think will happen if you do this?

5. Can you tell me what you know about this disease?

\section{Date analysis}

Data were simultaneously collected and analyzed. Within 48 hours after the interview, the recordings were transcribed word for word into written information, and data were analyzed by two people. Directed content analysis was used for data analysis. The goal of directed content analysis is to validate or extend a conceptual theoretical framework or theory [14]. The steps included (Table 2):

Table 2

Data analysis stages 


\begin{tabular}{|ll|}
\hline Stage & Description \\
\hline 2. preparation & $\begin{array}{l}\text { खRead the interview data repeatedly and get familiar with the content of the data to } \\
\text { get the overall sense of the data } \\
\text { खUnderline important, frequent statements that fit the purpose of this study }\end{array}$ \\
\hline $\begin{array}{l}\text { खUse underlined sentences as units of meaning, condense units of meaning, and } \\
\text { encode } \\
\text { खUsing deductive content analysis, similar codes are classified according to TPB to } \\
\text { form sub-themes, and sub-themes are summarized to form themes } \\
\text { खUsing inductive content analysis, similar codes that could not be grouped into }\end{array}$ \\
$\begin{array}{l}\text { TPB's original topic are grouped into new sub-themes, and sub-themes are } \\
\text { summarized to form themes }\end{array}$ \\
$\begin{array}{l}\text { Report the results according to the concept map, determine the corresponding } \\
\text { sample excerpt from the data, explain the results, and form the relationship between } \\
\text { the data and the theme }\end{array}$ \\
\hline
\end{tabular}

\section{Ethical consideration}

Consent for the study was obtained from the ethics committee of Daqing campus of Harbin Medical University(HMUDQ20211210003). All interviewees were informed about the objectives of the research, and informed written consent was obtained from all of them. We promised that the interview materials will be solely used for research purposes and will not include their personal and private information. Interviewees have the right to withdraw from the study at any stage.

\section{Consideration of rigor}

First, a research group (three nursing graduate students, one community nurse, one professor of nursing) was established, and a professor who engaged in qualitative research was invited to participate in the preparation of the interview outline. As a graduate student engaged in chronic disease health management, the researcher had 2 years of work experience in a large general hospital and received training in qualitative research and interview skills. A good communication relationship was established with the interviewees before the interview to gain the trust of the patients. After each interview, the data was fed back to the interviewees to check the authenticity of the data. Data analysis was conducted by two researchers simultaneously and supervised by professors familiar with the study.

\section{Results}

There were 20 participants in this study. The age of the participants was between 60 and 82 years old, and the mean age was 68.0 years. The disease course was between 1 and 15 years, the mean course of the disease was 6.3 years, the patient delay was between 0.6 and 10 years, and the mean patient delay 
was 3.52 years. There were 12 patients with urinary retention or hematuria, and 8 patients underwent surgery. All interviewees experienced patient delay. The interviewees' demographic characteristics are summarized in Table 3:

Table 3

Characteristics of participants

\begin{tabular}{|c|c|c|c|}
\hline Characteristic & Number & Characteristic & Number \\
\hline Age & & rural areas & 12 \\
\hline $60-69$ & 12 & urban areas & 8 \\
\hline $70-79$ & 7 & Monthly family income & \\
\hline$\geq 80$ & 1 & $\triangle 1000 \mathrm{CNY}$ & 12 \\
\hline Educational level & & 1000-2999 CNY & 5 \\
\hline Illiterate & 3 & 3000-4999 CNY & 2 \\
\hline primary school & 9 & $\geq 5000 \mathrm{CNY}$ & 1 \\
\hline junior high school & 4 & Patient delay『years】 & \\
\hline senior high school & 2 & $<1$ & 5 \\
\hline University & 2 & $1-5$ & 12 \\
\hline Marital status & & $\geq 5$ & 3 \\
\hline Married & 16 & IPSS凹score】 & \\
\hline death of spouse & 4 & $8-19$ & 13 \\
\hline Place of residence & & $20-35$ & 7 \\
\hline
\end{tabular}

Five main themes emerged from interviews. Notably, we expanded the TPB and established a new theoretical framework of intention and behavior of delay in seeking medical care in patients with BPH. In the theory of extended planned behavior(E-TPB), patient delay intention affects the patient delay behavior, and the patients' insufficient cognition of symptoms, negative attitude toward seeking medical care, the influence of others on decision-making for seeking medical care, previous experience with disease coping, and the degree of obstacles in seeking medical care affect the level of intention to delay seeking medical treatment. (Figure 1):

\section{Theme1: Insufficient cognition of symptoms}

\section{Low symptom alertness}


Almost all the interviewees could not detect early symptoms of BPH or felt that the symptoms were not serious and belittled the existence of the disease. (p3): "At the beginning, urination was not very smooth, and I felt that there was still urine after each urination, However, I didn't think the symptoms were very serious, so I still went to work as usual. I didn't think of going to the hospital for a check-up, and only went to see the doctor when I couldn't pee at all. If I had paid attention to these symptoms earlier, I wouldn't have to suffer such pain now."

\section{Symptom benign attribution}

Most interviewees did not understand the causes of BPH and mistakenly attributed LUTS to normal phenomena caused by age, weather changes, side effects of drugs, etc., which gave them a reasonable reason not to seek medical treatment. (P2): "I always think that this is not a disease, just old age. I am 66 now, my body is definitely not as good as when I was young, it's normal for me have a weak urinary stream, and even if you go to the hospital, you can't return to your youthful state!" (P1): "I need to take drugs for the treatment of high blood pressure for a long time. I think these drugs will stimulate the kidneys. My frequent urination is only a side effect of the drugs." (P6): "I used to think that nocturia was caused by the colder weather. As long as the weather gets warmer, the problem will get better, so I never thought about going to the hospital for an examination."

\section{Theme 2: Experience coping instead of going to a doctor}

Based on past experience with disease, a patient adopted the same approach when facing BPH, hoping to obtain the same effect as before. The interviewee said that in the early stage of illness, he would consider his previous personal experience, self-purchase medicine, and engage in self-conditioning instead of going to a doctor to deal with uncomfortable symptoms. (P5): "When I was sick before, I would observe the symptoms for a period of time, and then go to the pharmacy to buy some medicine. When I got this disease, I did the same. In fact, I also went to find folk prescriptions, hoping that it would make my illness better."

\section{Theme 3: Negative attitude toward seeking medical care}

\section{Underestimation of the consequences of delayed medical treatment}

Due to the atypical early symptoms of BPH and the slower progression of the disease, most interviewees paid low attention to the disease and underestimated the consequences of delayed medical treatment. (P7): "When I first got this disease, it didn't hurt or feel uncomfortable except for going to the toilet more times at night. I think maybe I don't need to go to the hospital, maybe the disease will heal automatically, or even if I go to the hospital later, it won't make the condition very serious." 


\section{Negative evaluation of early medical treatment}

Some interviewees had a negative evaluation of early medical treatment behavior. On the one hand, the reason was that they felt they could not benefit from early medical treatment due to repeated attacks and prolonged illness; on the other hand, they did not understand the characteristics of disease development and other medical treatment obstacles and had a negative attitude toward early medical treatment behavior. (P12): "This disease cannot be cured, no matter if you see a doctor early or late, it still cannot be cured, and the condition will always relapse." (P8): "When the condition is serious, you need to go to the hospital. As long as you can tolerate these symptoms, there is no need to go to the hospital. Besides, this is not a serious illness. Going to the hospital early will not only waste money but also delay your work."

\section{Theme 4: The influence of others on decision-making for seeking medical care}

\section{People who approve or disapprove of going to a doctor}

The interviewees reported that their family and friends had different opinions on whether to seek medical attention. When making medical decisions, the opinions of family and friends played a vital role. (P14): "My wife asked my son to take me to the hospital for an examination, so that they can know my health status, and if I get this disease, they suggest that I start treatment early." (P12): "Some friends around me have the same symptoms. They said that there is no need to go to the hospital. The man is old, and it is normal to have these symptoms. Just buy some medicine and take it. I think what they said should be correct."

\section{Do or do not follow the opinions of others}

Although some interviewees believed that family members' suggestions were important to their medical decision-making, there were still a few interviewees who said that they did not follow their family's expectations and did not choose to follow their suggestions. In addition, the interviewees believed that friends with the same disease had a substantial influence on their medical treatment behavior. If they chose to ignore the disease or buy medicines on their own, they were likely to adopt the same approach. (P10): "My daughter asks me to go to the hospital for a check-up. I always verbally agree to go, but I won't go there in the end." (P13): "Many old men around me have this disease, and they don't take it seriously, and they haven't been to the hospital. They said they just need to buy some medicine. When I get sick, I do the same as they do (buy the medicine by myself first)."

\section{Theme 5: Obstacles to seeking medical care}




\section{Factors from the patient}

Heavy financial burdens and fear of disrupting family life were the most frequently cited obstacles by the interviewees. (P8): "I rely on selling vegetables for a living. I work in the field every day. I can't spare time to go to the hospital, and the cost of seeing a doctor is also a lot. So, I decided not to go to the hospital, and wait until the symptoms became serious." (P9): "My son works in the city and he is very busy at work. I will not trouble him for this minor illness."

\section{Factors from medical institutions}

The quality of medical service and the intelligent medical treatment process affected the patient's medical decision-making. Three interviewees said they had bad medical experiences. (P15): "The doctor's level of care is very poor. I had a treatment there, and it relapsed in less than half a year, and it was more serious than before." (P13): "The registration is on the first floor, the doctor is on the fourth floor, and the check-up is in another building. It's too troublesome to run up and down by yourself. If it's not a serious illness, I don't want to go to the hospital." (P9): "To see a doctor in a large hospital, you need to register online, and you need to operate on the machine to get the inspection report, which is too difficult for me."

\section{Discussion}

This is the first qualitative study specifically aimed at exploring BPH patients' intention and behavior of patient delay. This study found that poor medical awareness and patient delay in patients with BPH are the result of a combination of many factors. From the interview data, we extracted 2 new themes outside of the TPB (insufficient cognition of symptoms and experience coping instead of going to a doctor) and extended the original theoretical framework, which may help to comprehensively describe the reason for $\mathrm{BPH}$ patients' intention and behavior of patient delay.

\section{High-level cognition of symptoms and a positive attitude toward medical treatment promote timely medical treatment}

The results of this study suggest that low-level or false cognition of symptoms and negative attitudes toward medical treatment can easily cause patients to have delayed intentions, leading to delayed patient behavior. First, LUTS caused by BPH lacks typical manifestations in the early stage, and the symptoms progress very slowly. A patient's long-term survival with the disease causes them to be less vigilant about the disease, and it is therefore easier to ignore the existence of the disease, which leads to patient delay. Second, BPH is prone to occur in the elderly, who often live alone and have multiple diseases. While their perception and judgment of the severity of symptoms are weakened, they are also subject to mistakenly attribute the symptoms to benign causes [15]. In this study, a total of 16 patients attributed LUTS to a 
normal phenomenon caused by aging rather than $\mathrm{BPH}$, gave a benign explanation to the symptoms, and refused to seek medical help. In contrast, correct symptom attribution may significantly improve delay by triggering more prompt and appropriate subsequent behaviors [15]. BPH patients' low alertness to symptoms, incorrect judgment of the condition, and benign attribution of symptoms are due to lack of cognition of LUTS. In fact, a lack of understanding of disease symptoms leading to patient delay is a common problem for most chronically ill patients [16]. In addition, the popularization of BPH education in China is far less than that of common chronic diseases such as hypertension and diabetes. The patient delay for BPH may be more prominent.

LUTS will slowly progress with the age of patients. The 2014 edition of the Chinese Urology Guidelines pointed out that the average annual increase in IPSS scores of patients with BPH ranges from 0.29 to 2 points. When the IPSS $\leq 7$, that is, when the quality of life of mild or asymptomatic patients is not affected, there is no need to seek medical treatment [17]. For patients with LUTS (IPSS $>7$ ), the purpose of early medical treatment and adherence to treatment is to relieve symptoms, reduce the risk of LUTS progression, reduce complications, and reduce surgery rates [18]. Short-term use of drug therapy is not significant for changing the clinical outcome, and this is likely to cause the early medical treatment effect to be lower than the patient's expectation and cause the patient to have a negative attitude toward medical treatment [19]. Low-level or false cognition of symptoms and negative attitudes toward medical treatment will inevitably affect patients' behavior in seeking medical care, which is consistent with the results of similar studies [16]. Expanding the individual's symptom cognitive reserve and correcting the negative attitude toward medical treatment are the first steps to promote timely medical treatment, especially focusing on the high-risk groups of BPH/LUTS.

\section{Role models and a good medical atmosphere guide correct medical behavior}

Advice from people considered to be important to the patient will influence individual medical decisions. The patient's family or friends have false cognition of symptoms and negative attitudes toward BPH, leading to incorrect medical advice provided to the patient and affecting the patient's subsequent health behavior. In addition, the medical treatment behavior of these important people will also indirectly affect the patient's medical decision-making. In the interviews, 12 rural patients said that their family members and friends had experienced patient delay, which made the patients mistakenly believe that patient delay is a normal behavior that is common in the process of medical treatment and has no effect on the condition. China's emphasis on BPH health management is also relatively weak. The public awareness of seeking medical treatment is generally low. Studies have shown that a good role model or a good medical treatment atmosphere can promote patients' health decisions, and the absence of these factors can induce patient delay [16]. Therefore, improving the medical awareness of patients, their families and friends, and the public, while ensuring that patients receive effective family and social support in guiding their correct medical behavior, are key points to improving patient delay in BPH. Studies have suggested that public health interventions (using TV commercials, radio broadcasts, etc.) have a certain effect on 
improving the public's knowledge of diseases [20]. Community medical staff can carry out similar publicity-type activities in the community (especially focusing on rural areas) improving public knowledge of BPH, correcting patients' incorrect experience behavior, promoting patients to seek timely medical treatment, and being role models.

\section{Optimizing medical services and reducing the burden of disease are common needs}

This study suggests that the quality of medical services and cumbersome medical procedures are factors that cause patient delay, which is consistent with the results of previous studies [16, 21]. The delay in rural areas is more serious, which is related to the remoteness of rural residents, lack of concentrated housing, and lack of human resources [22]. During the interview, a rural patient reported that his condition was delayed because the doctor did not have enough clinical experience and ability. There are abundant medical resources in urban areas, but intelligent medical treatment processes such as online appointment registration, mobile payment, and online examination reports, are not feasible for most elderly people. In addition, economic burden is an important obstacle in patients not going to see a doctor. Research statistics reveal that the average hospitalization cost of BPH inpatients (873 cases) is CNY $21,221.48$, of which the largest proportion is the cost of medicine, followed by the cost of surgery, anesthesia, and inspection [23]. This will undoubtedly cause a heavy economic burden on unemployed elderly individuals. Enriching medical and health service resources in rural areas, reforming the medical treatment process to adapt to aging, and seeking complete BPH medical insurance are common demands of patients.

\section{Conclusions}

The results of this study showed that the average patient delay for 20 patients was 3.52 years, and the delay was longer in rural areas than in urban areas. Insufficient cognition of symptoms, incorrect empirical behavior, and heavy financial burden were the main factors that affect BPH patients' intention and behavior of patient delay.

\section{Limitations}

First, data collection was only from the patient. In future research, interaction with the patient's family and community hospital can be increased to establish a multi-dimensional descriptive database. Second, due to the cultural differences between English and Chinese, the transcription of interview information is also a limitation of this research. Finally, although we expanded the TPB and established a new theoretical framework, the relationship between variables still needs to be verified by subsequent quantitative studies to prove the effectiveness of E-TPB.

\section{Abbreviations}


BPH: Benign Prostatic Hyperplasia; LUTS: Lower Urinary Tract Symptoms; IPSS: International Prostate Symptom Score; TPB: Theory Of Planned Behavior; CNY: China Yuan; P1: Participant 1

\section{Declarations}

\section{Acknowledgements}

We would like to thank the 20 participants for sharing their experience of patient delay. We would also like to thank Wanbao Community Hospital for providing us with a free interview room.

\section{Authors' contributions}

$\mathrm{ZH}, \mathrm{JDX}$ and ZLL contributed the study design, conducted the interviews, analyzed the data, and led the drafting of the manuscript. ZWY, YJH, JHH and LYL contributed to draft a interview outline. All authors read and approved the final manuscript.

\section{Funding}

The study was financially supported by the Natural Science Foundation of Heilongjiang Province (LH2019G011)

\section{Availability of data and materials}

The data are not publicly available due to them containing information that could compromise research participant privacy. but are available from the corresponding author on reasonable request.

\section{Ethics approval and consent to participate}

The study was approved by the ethics committee of Daqing campus of Harbin Medical University(HMUDQ20211210003) in accordance with the guidelines of the Declaration of Helsinki. All interviewees were informed about the objectives of the research, and informed written consent was obtained from all of them and also from the legal guardians of the illiterate participants.

\section{Consent for publication}

Not applicable.

\section{Competing interests}


The authors declare that they have no competing interests.

\section{Author details}

${ }^{1}$ Department of Nursing, Harbin Medical University (Daqing), 163319, China.

2Urology, People's Hospital in Hei Longjiang Province, Daqing, 163319, China.

${ }^{3}$ Department of Nursing, Changzhou University, Jiangsu, 213164, China. zhanglinlin1971@163.com

\section{References}

1. Lee S, Chan E, Lai YK. The global burden of lower urinary tract symptoms suggestive of benign prostatic hyperplasia: A systematic review and meta-analysis. Sci Rep. 2017-08-11 2017;7(1):7984.

2. Hu Yongtao,Zou Zhihui,Liang Chaochao.New advances in surgical treatment of benign prostatic hyperplasia. Chinese Journal of Endourology(Electronic Edition).2021;15(01):84-88.

3. Egan KB. The Epidemiology of Benign Prostatic Hyperplasia Associated with Lower Urinary Tract Symptoms: Prevalence and Incident Rates. Urol Clin North Am. 2016-08-01 2016;43(3):289-297.

4. Wang Guangrong, Chen Jianwen, Li Xueyan.The willingness and influencing factors of elderly patients with benign prostatic hyperplasia in the community. Chinese Journal of Gerontology. 2016;36(06):1465-1467.

5. Roghmann F, Ghani KR, Kowalczyk KJ, et al. Incidence and treatment patterns in males presenting with lower urinary tract symptoms to the emergency department in the United States. J Urol. 2013-1101 2013;190(5):1798-1804.

6. Speakman M, Kirby R, Doyle S, loannou C. Burden of male lower urinary tract symptoms (LUTS) suggestive of benign prostatic hyperplasia (BPH) - focus on the UK. BJU INT. 2015-04-01 2015;115(4):508-519.

7. Pack GT, Gallo JS. The culpability for delay in the treatment of cancer. American Journal of Cancer. 1938;33.

8. Lin CY, Oveisi S, Burri A, Pakpour AH. Theory of Planned Behavior including self-stigma and perceived barriers explain help-seeking behavior for sexual problems in Iranian women suffering from epilepsy. EPILEPSY BEHAV. 2017-03-01 2017;68: 123-128.

9. Lin CY, Broström A, Årestedt K, Mårtensson J, Steinke EE, Pakpour AH. Using extended theory of planned behavior to determine factors associated with help-seeking behavior of sexual problems in women with heart failure: a longitudinal study. J Psychosom Obstet Gynaecol. 2020-03-01 2020;41(1):54-61.

10. Mak HW, Davis JM. The application of the theory of planned behavior to help-seeking intention in a Chinese society. Soc Psychiatry Psychiatr Epidemiol. 2014-09-01 2014;49(9):1501-1515. 
11. Ajzen I. From Intentions to Actions: A Theory of Planned Behavior[J]. Advances in Experimental Social Psychology, 1987, 20(8):1-63.

12. Ajzen I. Constructing a TPB questionnaire: Conceptual and methodological considerations. accessed 28/12/2007. 2002.https://people.umass,edu/aizen/pdf/tpb.measurement.pdf.

13. Shi W, Hall BJ. Help-seeking intention among Chinese college students exposed to a natural disaster: an application of an extended theory of planned behavior (E-TPB). Soc Psychiatry Psychiatr Epidemiol. 2021-07-01 2021;56(7):1273-1282.

14. Sabzmakan L, Eslami F, Sighaldeh SS, Izuka NJ. Intention to quit water pipe smoking among Iranian women: a qualitative directed content analysis. BMC WOMENS HEALTH. 2020-03-23 2020;20(1):59.

15. McKee G, Mooney M, O'Donnell S, O'Brien F, Biddle MJ, Moser DK. Multivariate analysis of predictors of pre-hospital delay in acute coronary syndrome. INT J CARDIOL. 2013-10-03 2013;168(3):27062713.

16. Jia HH, Liu L, Huo GX, Wang RQ, Zhou YQ, Yang LY. A qualitative study of the cognitive behavioral intention of patients with diabetes in rural China who have experienced delayed diagnosis and treatment. BMC PUBLIC HEALTH. 2020-04-10 2020;20(1):478.

17. Mobley D, Feibus A, Baum N. Benign prostatic hyperplasia and urinary symptoms: Evaluation and treatment. POSTGRAD MED. 2015-04-01 2015;127(3):301-307.

18. Nickel JC. The economics of medical therapy for lower urinary tract symptoms associated with benign prostatic hyperplasia. CURR UROL REP. 2006-07-01 2006;7(4):282-287.

19. Cindolo L, Pirozzi L, Fanizza C, et al. Drug adherence and clinical outcomes for patients under pharmacological therapy for lower urinary tract symptoms related to benign prostatic hyperplasia: population-based cohort study. EUR UROL. 2015-09-01 2015;68(3):418-425.

20. Baker $\mathrm{H}$, Murdoch IE. Can a public health intervention improve awareness and health-seeking behaviour for glaucoma? Br J Ophthalmol. 2008-12-01 2008;92(12):1671-1675.

21. Tong $Y$, Guan X, Hou S, et al. Determinants of Health Care-Seeking Delay among Tuberculosis Patients in Rural Area of Central China. Int J Environ Res Public Health. 2018-09-13 2018;15(9).

22. Chen Mo. Analysis of the Predicament of Medical Services in Rural China and Countermeasures Assisted by Artificial Intelligence. Chinese Health Service Management. 2021;38(07):536-538.

23. Xie Wei. Analysis on the hospitalization Expenses of Benign Prostatis Hypertrophy Medicare Inpatients with Factors Analysis. Chinese Medical Record.2017;18(11):54-56.

\section{Figures}




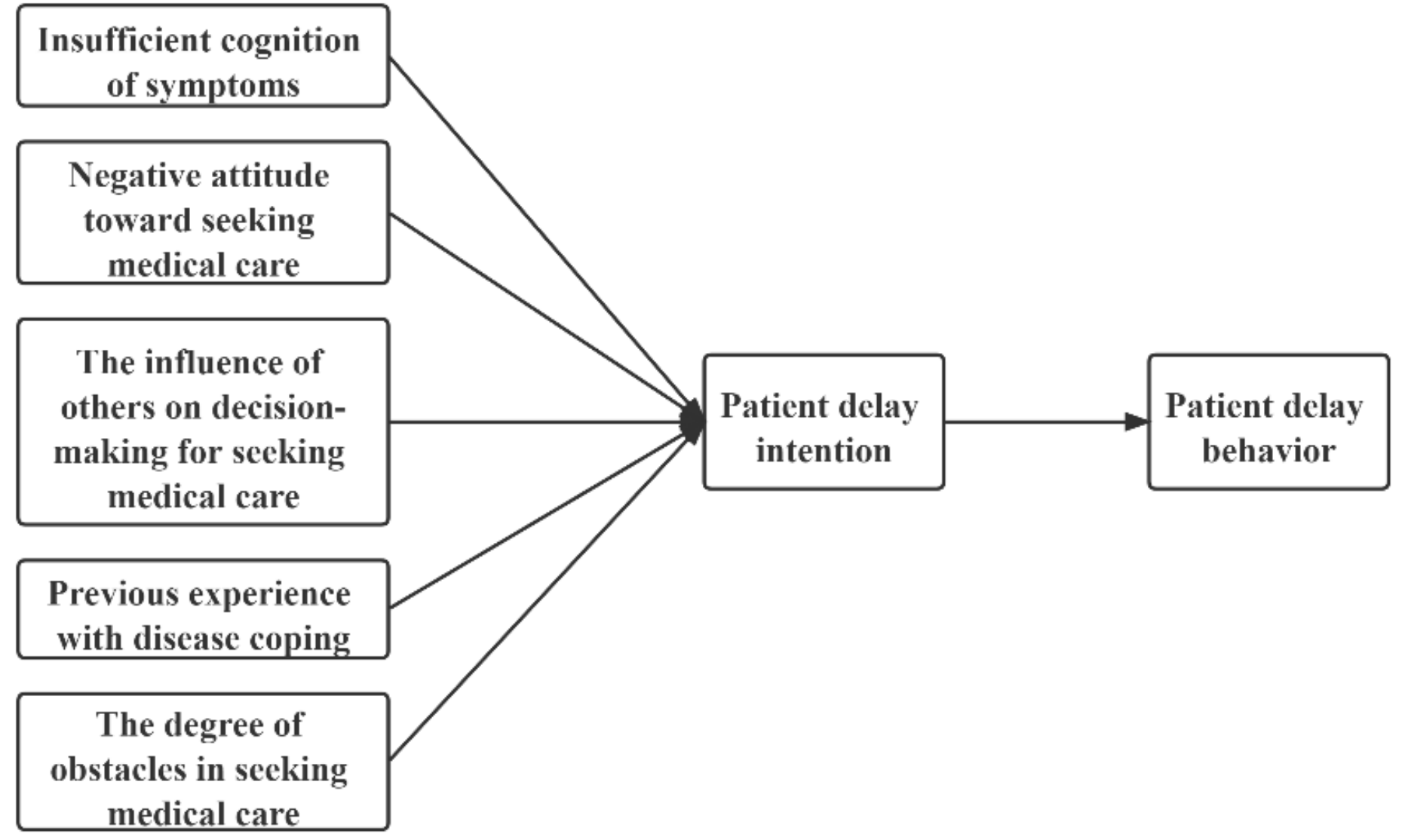

Figure 1

Extended theory of planned behavior(E-TPB) 